\title{
Obstrucción mecánica de colon producida por un catéter autoposicionante de diálisis peritoneal
}

\author{
Beatriz Peláez Requejoํㅗㄹ Mónica Fernández Pérez, Isabel González Díaz, Reyes Fernández Díaz¹, Miguel Núñez \\ Moral $^{1}$, Aurora Quintana Fernández \\ Unidad de Diálisis Peritoneal. Unidad de Gestión Clínica de Nefrología. HUCA. Oviedo. España \\ ${ }^{1}$ Instituto de Investigación Sanitaria del Principado de Asturias (ISPA)
}

\section{Resumen}

Introducción: La migración del catéter de diálisis peritoneal constituye una complicación que condiciona la viabilidad de la técnica, por eso para tratar de disminuir las disfunciones por desplazamiento apareció en 1996 el catéter Autoposicionante.

Caso clínico: Presentamos el caso de un varón portador de catéter Swan Neck al que se realizan 2 maniobras alfa para intentar corregir desplazamiento precoz y que finalmente precisa recambio quirúrgico, implantándose en esta ocasión catéter Autoposicionante.

Durante el periodo de cicatrización presenta nuevo episodio de migración pero se consigue reposicionamiento con alambre guía y es posible reiniciar la técnica.

Solo tres meses después necesita ingreso hospitalario por clínica abdominal y los estudios de imagen demuestran dilatación de ciego y colon hasta el cruce con el catéter de diálisis peritoneal, llegándose al diagnóstico de obstrucción mecánica de colon por catéter de diálisis.

Esta complicación grave apareció en contra de las expectativas planteadas basándonos en nuestra propia experiencia y los resultados de múltiples estudios.

Correspondencia:

Beatriz Peláez Requejo.

Unidad de Diálisis Peritoneal. UGC de Nefrología

Hospital Universitario Central de Asturias

C/Roma s/n. 33011 Oviedo

E-mail: beatrizpelaezr@yahoo.es
Palabras Clave: diálisis peritoneal; catéter autoposicionante; migración de cuerpo extraño; complicaciones.

\section{Colon mechanical obstruction produced by a self-locating catheter of peritoneal dialysis}

\section{Abstract}

Introduction: The migration of the peritoneal dialysis catheter constitutes a complication that conditions the viability of the technique, so in order to try to reduce dysfunctions due to displacement, the self-locating catheter appeared in 1996.

Case report: We present the case of a male, Swan Neck catheter carrier, whom 2 alpha maneuvers to try to correct early displacement were developed and finally surgical replacement was required, implanting a self-locating catheter.

During the healing period, a new episode of migration appears, but repositioning with guide wire is achieved and it is possible to restart the technique.

Only three months later, he needed hospital admission for abdominal surgery and the imaging studies showed dilation of the caecum and colon until the junction with the peritoneal dialysis catheter, being diagnosed of coIon mechanical obstruction produced by dialysis catheter. 
This serious complication appeared, contrary to the expectations raised, based on our own experience and the results of multiple studies.

Keywords: peritoneal dialysis; self-locating catheter; foreign body migration; complications.

\section{Introducción}

El catéter peritoneal resulta imprescindible para la realización de la diálisis peritoneal como técnica de terapia renal sustitutiva y es una preocupación desde sus inicios el encontrar el acceso peritoneal ideal.

A lo largo del tiempo se han desarrollado distintos modelos, la mayoría de ellos modificaciones del catéter Tenckhoff, que han conseguido hacer de la Diálisis Peritoneal una técnica continua y ambulatoria ${ }^{1,2}$.

En 1996 apareció el catéter Autoposicionante de Di Paolo con la intención de disminuir las disfunciones por desplazamiento gracias al peso de tungsteno de $12 \mathrm{~g}$ de su extremo distal que lo ayuda a permanecer en su posición en fondo de saco de Douglas 3 .

El desplazamiento del catéter condiciona su funcionamiento, y por consiguiente la viabilidad de la técnica, y está íntimamente ligado al ritmo intestinal. La solución a esta complicación que representa aproximadamente el $20 \%$ de las transferencias a Hemodiálisis ${ }^{4}$, pasa en primer lugar por aumentar la peristalsis con enemas de limpieza o la toma de soluciones evacuantes. Si esto no resulta efectivo se recurre a la movilización del catéter mediante guía, conocida como maniobra alfa (técnica realizada por el Servicio de Radiología Vascular Intervencionista) y en último lugar a la recolocación quirúrgica o el reemplazo del mismo ${ }^{5}$.

\section{Caso clínico}

Presentamos el caso de un varón de 55 años, $91 \mathrm{Kg}$ de peso y $187 \mathrm{~cm}$ de talla (Índice Masa Corporal 26). EI diagnóstico de base es Enfermedad Renal Crónica secundaria a glomerulonefritis mesangial IgA.

Es remitido a nuestra Unidad tras optar por la diálisis peritoneal como terapia renal sustitutiva. El día 19/02/2016 se coloca mediante cirugía un catéter Swan Neck Fast Flow cuello cisne, doble cuff y punta espiral con funcionamiento inicial bueno.
Tan sólo un mes después, todavía durante el periodo de entrenamiento, se identifica drenaje lento e incompleto y se objetiva desplazamiento del catéter hacia mesogastrio en una radiografía simple de abdomen (Figura 1). Se indica enema de limpieza y toma de laxante con macrogol según protocolo de la Unidad.

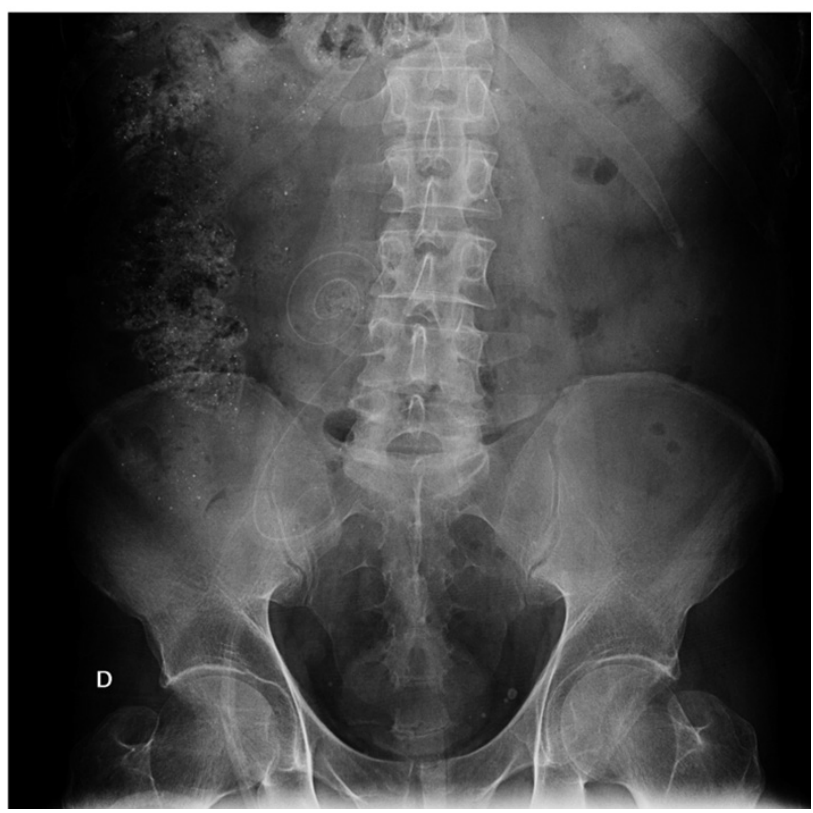

Figura 1.

Dada la ineficacia de las medidas, se realiza la primera maniobra alfa que, a pesar de conseguir mínimo cambio de posición de la vuelta distal, mejora su funcionamiento y permite el inicio de la técnica en domicilio, primero en Diálisis Peritoneal Continua Ambulatoria y posteriormente, respetando la preferencia del paciente, inicia Diálisis Peritoneal Automatizada, pero los drenajes son dificultosos, así que precisa una segunda maniobra alfa que no consigue el reposicionamiento del catéter que permanece en flanco derecho.

Se decide de acuerdo con el paciente el recambio del catéter, implantando en esta ocasión un catéter Autoposicionante, Care-Cath, doble cuff y cuello recto, en hemiabdomen izdo el 31/05/2016.

Durante el tiempo de cicatrización, el paciente se mantiene con tratamiento conservador y ya presenta dolor y desplazamiento hacia flanco izquierdo, con el extremo distal enfocado hacia arriba (Figura 2); no se consigue movilizar con las medidas habituales y realiza la tercera maniobra alfa, siendo ésta efectiva y reinicia tratamiento nocturno con la cicladora. 
A los tres meses acude a nuestra Unidad con temperatura de $38^{\circ} \mathrm{C}$, sin clínica abdominal y analítica de sangre y líquido peritoneal normales, se diagnostica de crisis de podagra y se inicia tratamiento farmacológico.
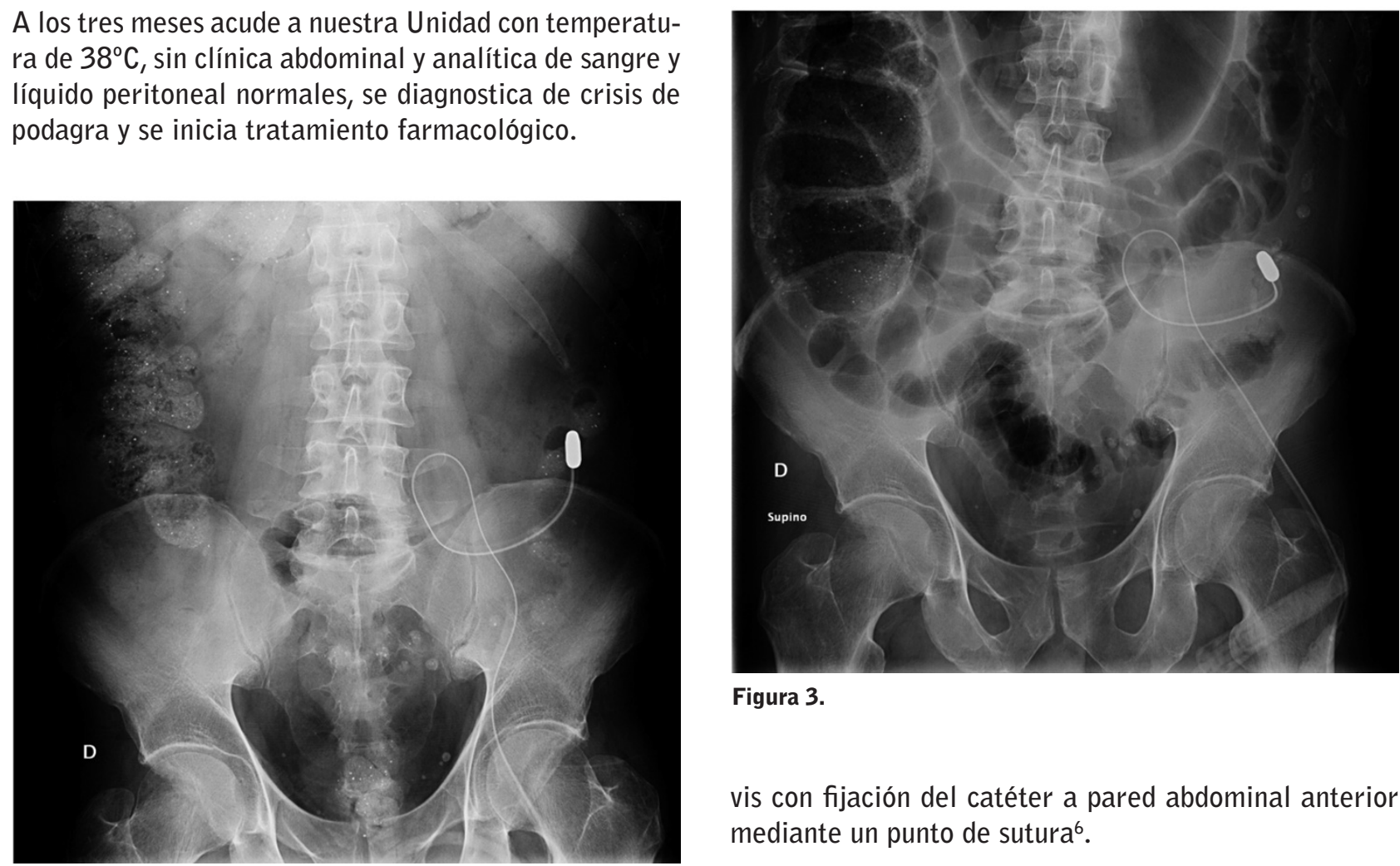

Figura 3.

vis con fijación del catéter a pared abdominal anterior mediante un punto de sutura ${ }^{6}$.

Figura 2.

A los tres días de evolución hay empeoramiento clínico, con dolor abdominal difuso, persistencia de febrícula y sin alteraciones del ritmo intestinal referidas; los parámetros analíticos se mantienen dentro de la normalidad pero en la radiografía de abdomen se aprecian abundantes heces y el catéter de diálisis de nuevo en flanco izquierdo con el extremo distal hacia arriba.

Pasadas 24 horas ingresa a cargo del Servicio de Nefrología para estudios por clínica abdominal. El abdomen está ahora timpánico y distendido, la ecografía inicial es normal y en la radiografía de abdomen se observa dilatación de asas (Figura 3); es valorado por el Servicio de Cirugía General y se trata como una suboclusión. La mala evolución indica TAC urgente que muestra dilatación de ciego de $65 \mathrm{~mm}$ de diámetro, moderada distensión del colon ascendente y transverso que disminuye progresivamente en colon descendente hasta el cruce con el catéter de diálisis peritoneal en la unión colosigmoidea, llegándose al diagnóstico de obstrucción mecánica de colon por catéter de diálisis.

Se realiza laparoscopia que evidencia punta de catéter en mesocolon izquierdo, se extrae y se coloca en la pel-

Tras la cirugía el paciente evoluciona favorablemente pero la sujeción del catéter obliga a la diálisis manual por dificultad en los drenajes.

Tres meses más tarde el paciente recibe Trasplante renal de donante cadáver y se retira el catéter de diálisis peritoneal en el mismo acto quirúrgico.

Presentamos este caso al considerarlo de interés, ya que a pesar de que diversos estudios $7,8,9,10$ relacionan el catéter autoposicionante con una menor tasa de migraciones, pudiéndose corregir con más facilidad que con otro tipo de catéteres si se llegan a producir ${ }^{11}$, en este caso hemos podido comprobar que no se han cumplido las expectativas que se tenían al implantarlo, ya que se han repetido episodios de migración del catéter, y además se ha originado una complicación grave como la obstrucción intestinal, no descrita con anterioridad en la literatura revisada ${ }^{12}$.

\section{Recibido: 23 abril 2018}

Revisado: 10 octubre 2018

Modificado: 20 noviembre 2018

Aceptado: 23 noviembre 2018 


\section{Bibliografía}

1. Twardowski ZJ. History of peritoneal access development. Int J Artif Organs 2006;29:2-40.

2. Dell'Aquila R; Chiaramonte S; Rodighiero MP; Spano E; Di Loreto P; Ocampo Kohn C el all. Rational choise of peritoneal dialisys catheter. Perit Dial Int 2007;27(S2):S119-25.

3. Di Paolo N; Petrini G; Garosi G; Buoncristinati U; Brardi S; Monaci G. A new self- locating peritoneal catéter. Perit Dial Int 1996;16:623-7.

4. Mc Cormick B; Bargman J. Noninfectious Complications of Peritoneal Dialisys: Implications for Patient and Technique Survival. J Am Soc Nephrol 2007;18:3023-5.

5. Rodríguez-Palomares JR; Ruiz C; Granado A; MontenegroJ. El acceso peritoneal. Complicaciones no infecciosas del acceso peritoneal. Guías de práctica clínica en diálisis peritoneal. Nefrología 2006; 26:99-107.

6. Kume $\mathrm{H}$; Miyazaki $\mathrm{H}$; Nagata $\mathrm{M}$; Ishikawa $A$; Ishibashi $Y ;$ Fujita $T$ et al. Peritoneal fixation prevents dislocation of Tenckhoff catheter. Perit Dial Int 2011;31:694-7.
7. Sánchez JJ; García H; García R; Pascual MJ; Casado $\mathrm{D}$. Prospective randomized study comparing a single-cuff self-locating catheter with a single-cuff straight Tenckhoff catheter in peritoneal dialisys. Perit Dial Int 2016;36:52-9.

8. Salvador MC; Folch MJ; Mallol A; Bort J; Renau $\mathrm{E}$; Cerrillo $\mathrm{V}$ el all. Estudio comparativo entre catéteres peritoneales autoposicionantes y catéteres Tenckhoff en referencia a su desplazamiento intraperitoneal. Enferm Nefrol 2012;15:188-93.

9. Di Paolo N; Capotondo L; Brardi S; Nicolai G. The selflocating peritoneal catheter: fifteen years of experience. Perit Dial Int 2010;30:504-5.

10. Bergamin B; Senn 0; Corsenca A; Dutkowski P; Weber $\mathrm{M}$; Wüthrich RP et al. Finding the right position: a three-year, single-center experience with the "self-locating" catheter. Perit Dial Int 2010;30:519-23.

11. Di Paolo N; Capotondo L; Sansoni E; Romolini V. The self-locating catheter: clinical experience and follow-up. Perit Dial Int 2004;24:359-64.

12. Moreiras M; Martín IM; Blanco R; Beato LV; Cossio CR. New Peritoneal Catheters: New Catheter Problems?. Perit Dial Int 2014;34:556-61.

Este artículo se distribuye bajo una Licencia Creative Commons Atribución-NoComercial 4.0 Internacional. https://creativecommons.org/licenses/by-nc/4.0/ 